
\section{ทุอฝำ}

\section{Pamifos $^{\circledR}$ - Die neue Lösung von medac}

Bisphosphonate sind potente Hemmer der Osteoklasten-induzierten Knochenresorption. Sie sind effektiv in der Therapie von Knochenmetastasen und anderen krebsbedingten Knochenkomplikationen, indem sie das Skelett vor weiterer Zerstörung schützen, die Schmerzen effektiv lindern und signifikant die Lebensqualität erhöhen. So kann eine monatliche Infusion von $\mathrm{Pa}$ midronsäure (Pamifos ${ }^{\circledR}$ ) Frauen mit fortgeschrittenem Brustkrebs vor Komplikatio-

\section{VELCADE Bortezomib}

\section{Proteasom-Inhibition in der Mye- Iomtherapie mit Velcade ${ }^{\circledR}$ : Bortezo- mib verlängert krankheitsfreie Zeit}

Für die Therapie des multiplen Myeloms ist seit Mai 2004 ein neuer Wirkstoff verfügbar, der auf dem Prinzip der Proteasom-Inhibition beruht und einen Durchbruch in der Behandlung des therapieresistenten multiplen Myeloms darstellt. Bortezomib (Velcade ${ }^{\circledR}$, Ortho Biotech, Geschäftsbereich von Janssen-Cilag) bewirkt bei austherapierten Patienten eine Ansprechrate von $35 \%$, verlängert die krankheitsfreie nen schützen. Auch bei Patienten mit multiplem Myelom zeigt Pamidronsäure prophylaktische Wirkung.

Die medac $\mathrm{GmbH}$ hat ihr Sortiment zum 15. Juni 2004 um ein weiteres Bisphosphonat erweitert und unter dem Handelsnamen Pamifos ein Pamidronat-Konzentrat zur Herstellung einer Infusionslösung auf den Markt gebracht. Zugelassen ist Pamifos ${ }^{\circledR}$ für die Therapie der tumorinduzierten Hyperkalzämie, osteolytische Läsionen bei Patienten mit Knochenmetastasen infolge eines Mammakarzinoms und beim Multiplen Myelom. Die Pamifos-Lösung kann nach Verdünnung sofort infundiert werden und ist über 96 Stunden physikalisch-chemisch stabil.

Das Konzentrat in der Durchstechflasche aus Duranglas steht in den Wirkstärken 15,
30, 60 und $90 \mathrm{mg}$ zur Verfügung, die in drei Packungsgrößen mit 1, 4 und 10 Durchstechflaschen erhältlich sind.

Medac bietet als exklusiven Service ergänzend zu Pamifos die Surefuser ${ }^{\circledR}$-Pumpe für eine 2-Stundeninfusion an. Diese mobile Lösung spart Zeit und Kosten in Praxis und Apotheke und erhöht die Lebensqualität des Patienten durch Erhaltung maximaler Beweglichkeit.

Weitere Informationen bei:

Dr. Frank Künkel

Medac GmbH

Supportiva Onkologie

Tel. +49 410380 06-4 383, Fax -100

E-mail f.kuenkel@medac.de

www.medac.de
Zeit im Vergleich zur letzten Vortherapie von 3 auf 7 Monate und erreicht ein Gesamtüberleben von 17,5 Monaten, wie die Zulassungsstudie SUMMIT (Phase II) gezeigt hat. Auf dem 9. Kongress der Europäischen Hämatologie-Gesellschaft in Genf wurden im Juni Zwischenergebnisse einer Phase-III-Studie präsentiert, die die positive Datenlage von Bortezomib bestätigen. Prof. Dr. Jesús San Miguel, Salamanca/Spanien, erläuterte die Daten: «Die Zwischenanalyse hatte unter Bortezomib eine deutliche Verlängerung der Zeit bis zum Fortschreiten der Erkrankung (TTP) um 58\% ergeben: Sie betrug unter Bortezomib im Schnitt 5,7 Monate, unter Dexamethason hingegen 3,6 Monate. Zudem verringerte sich für die Patienten unter Bortezomib die Wahrscheinlichkeit, während des ersten Jahres zu versterben, um etwa $30 \%$. Dies fand unabhängig davon statt, dass etwa
$50 \%$ der Patienten, die mit Dexamethason behandelt wurden, aufgrund der signifikant besseren Ergebnisse in der BortezomibGruppe die Therapie wechselten.» Ein weiteres überzeugendes Resultat: Die Infektionsrate Grad 3 und höher lag im Bortezomib-Arm bei $6,7 \%$, im DexamethasonArm hingegen bei 10,6\%. Dies ist die erste randomisierte Studie beim rezidivierten multiplen Myelom, die einen Überlebensvorteil im Vergleich zu einer Standardtherapie gezeigt hat.

In laufenden zusätzlichen Analysen werden derzeit weitere Parameter wie Ansprechen, Dauer des Ansprechens, Überleben und Sicherheit untersucht.

Weitere Informationen bei:

Kerstin Aschoff

Janssen-Cilag GmbH

Tel. +49 2137 955-412

Email kaschoff@jacde.jnj.com

\title{
PharmaTicker+++ PharmaTicker+++ PharmaTicker+++ PharmaTicker+++
}

Novartis Pharma GmbH. Femara ${ }^{\circledR}$ (Letrozol) hat über ein beschleunigtes Verfahren in der Schweiz die Zulassung erhalten für die erweiterte adjuvante Behandlung von Frauen nach der Menopause mit Hormonrezeptor-positivem oder Hormonrezeptor-unbekanntem Brustkrebs im Frühstadium, die postoperativ eine 5 Jahre dauernde Tamoxifentherapie erhalten haben. «Erweitert adjuvant» bezieht sich auf den Zeitraum im Anschluss an die Standardbehandlung. Weitere Informationen bei:

Novartis Pharma GmbH, Philipp Kreßirer

Tel. + 49 911 273-12006, Fax -12971

E-mail philipp.kressirer@pharma.novartis.com
Bayer HealthCare AG. Das neue Krebsmedikament BAY43-9006 hat den Orphan-Drug-Status zur Behandlung von fortgeschrittenen Nierenzellkarzinomen erhalten. Die Europäische Kommission gewährt diesen Status für Medikamente, die zur Behandlung seltener Krankheiten geeignet sind. Die neue Substanz wird zurzeit in der Behandlung von metastasierendem Nierenzellkarzinom im Rahmen einer weltweiten Phase-III-Studie untersucht.

Weitere Informationen bei:

Bayer Health Care AG, Hartmut Alsfasser Tel. +49 214 30-57526

E-mail hartmut.alsfasser@bayerhealthcare.com
Hoffman-La Roche AG. Roche hat bei den europäischen und US-amerikanischen Gesundheitsbehörden den Antrag zur Zulassung von Capecitabin $\left(\mathrm{Xeloda}^{\circledR}\right.$ ) für die adjuvante $\mathrm{Be}$ handlung des Kolonkarzinoms eingereicht. Der Antrag stützt sich auf Ergebnisse der X-ACTStudie, die zeigt, dass das orale Fluoropyrmidin Capecitabin mindestens genauso wirksam und besser verträglich ist als das intravenöse MayoKlinik-Protokoll. Capecitabin könnte damit die intravenöse 5-FU/LV-Gabe ersetzen.

Weitere Informationen bei:

Hoffmann-LaRoche AG, Dr. med. H.-U. Jelitto

Tel. +49 7624 14-2400, Fax -3366

\begin{tabular}{ll}
\hline KARGER & ( 2004 S. Karger GmbH, Freiburg \\
Fax + 49 76145207144 & $\begin{array}{l}\text { Accessible online at: } \\
\text { www.karger.com/onk }\end{array}$
\end{tabular}




\section{Chemotherapie des Prostata- karzinoms}

Taxotere ${ }^{\circledR}$ ist die erste und einzige Chemotherapie mit nachgewiesenem signifikantem Überlebensvorteil für Männer mit hormonrefraktärem, metastasiertem Prostatakrebs. Im Mai 2004 erteilte die amerikanische Arzneimittel behörde FDA die Zulassung für Taxotere zum Einsatz bei hormonrefraktärem, metastasiertem Prostatakrebs. Die Zulassung für Europa wird Ende 2004 erwartet.

In der Plenarsitzung der Jahrestagung der American Society of Clinical Oncology (ASCO) wurden im Juni 2004 die Ergebnisse von zwei randomisierten Phase-IIIStudien mit Taxotere (Wirkstoff: Docetaxel) zur Behandlung des hormonrefraktären, metastasierten Prostatakrebs vorgestellt. Die klinischen Studien TAX 327 und SWOG 9916 wurden von Prüfärzten des Johns Hopkins Kimmel Cancer Center, des Columbia Presbyterian Medical Center und der Southwest Oncology Group (SWOG) geleitet. Die Studien, an denen insgesamt etwa 1.800 Patienten teilnahmen, zeigen, dass Patienten durch die Behandlung mit Taxotere im Median 2,4 ( $\mathrm{p}=$ $0,009)$ bzw. 2 Monate $(p=0,01)$ länger leben als im Mitoxantron-haltigen Vergleichsarm. Darüber hinaus konnten durch den Einsatz von Taxotere im Vergleich zur Standardbehandlung (Mitoxantron) das Tumoransprechen (PSA-Abfall) der $\mathrm{Pa}-$ tienten signifikant um $43 \%(\mathrm{p}=0,0005)$ und tumorbedingte Schmerzen gar um $59 \%(p=0,0107)$ verbessert werden. Die Prüfer der SWOG-Studie berichteten von einem Anstieg des progressionsfreien Überlebens (PFS) um 27\%, einem Anstieg der Tumoransprechrate um $85 \%$ und einer Zunahme der objektiven Ansprechrate um
$55 \%$ im TaxotereArm der Studie. Zusätzlich konnte bei der Mehrheit der Patienten durch Taxotere ein Abfall im PSA-Wert erzielt werden.

\section{TAX 327/SWOG \\ 9916 Studien- protokoll}

An der Studie TAX 327 (Abb.), die von Mario Eisenberger, Dale Hughes Professor of Oncology and Urology am Johns Hopkins Kimmel Cancer Center, vorgestellt wurde, waren 1.006 Patienten an 240 Zentren in 24 Ländern beteiligt. Die Patienten wurden in eine von drei Behandlungsgruppen randomisiert. Taxotere $75 \mathrm{mg} / \mathrm{m}^{2}$ einmal alle 3 Wochen plus täglich Prednison oder Taxotere $30 \mathrm{mg} / \mathrm{m}^{2}$ jede Woche in 5 von 6 Wochen plus täglich Prednison oder Mitoxantron $12 \mathrm{mg} / \mathrm{m}^{2}$ alle 3 Wochen plus täglich Prednison, die etablierte Standardtherapie. Die meisten Patienten waren über 65 Jahre alt.

Bei der von Dr. Petrylak vorgestellten Studie SWOG 9916 wurden 770 Patienten in den USA in einen von zwei Behandlungsarmen randomisiert: Taxotere $60 \mathrm{mg} / \mathrm{m}^{2}$ alle 3 Wochen und Estramustin $280 \mathrm{mg} 3 \times$ täglich für 5 Tage oder Mitoxantron 12 $\mathrm{mg} / \mathrm{m}^{2}$ alle 3 Wochen und Prednison $5 \mathrm{mg}$ $2 \times$ täglich. In beiden Studien wurde Taxotere gut vertragen und hatte insgesamt ein kontrollierbares Nebenwirkungsprofil. Die am häufigsten beobachteten Nebenwirkungen innerhalb der TAX 327-Studie waren Haarausfall, Müdigkeit und Übelkeit. Die Häufigkeit des Auftretens war mit Mitoxantron vergleichbar. Die in der Studie beobachteten hämatologischen Ereignisse entsprachen bei beiden Therapien dem erwarteten Profil, wobei Grad-3-4-Neutropenie in der Taxotere-Gruppe häufiger auftrat als unter Mitoxantron (32 vs 21,7\%, $\mathrm{p}=0,004)$. Neutropeniebedingte Komplikationen waren jedoch in beiden Gruppen jeweils vergleichbar. In der Studie SWOG 9916 traten gastrointestinale und kardiovaskuläre Ereignisse häufiger bei den mit Taxotere plus Estramustin behandelten Männern auf als im Vergleichsarm.

Weitere Informationen bei:

Dr. Brigitte Hanke

Produktkommunikation Onkologie

Tel. +49 69 305-28183, Fax -28304

E-mail Brigitte.Hanke@aventis.com

\section{PharmaTicker+++ PharmaTicker+++ PharmaTicker+++ PharmaTicker+++}

Astra Zeneca GmbH. Zoladex ${ }^{\circledR}$ Gyn (Wirkstoff Goserelin) war bisher für die Behandlung von benignen hormonabhängigen Erkrankungen der Frau wie Endometriose und Myome der Gebärmutter zugelassen. Die Zulassung für ZoladexGyn wurde jetzt auf die Behandlung des hormonabhängigen Mammakarzinoms erweitert. ZoladexGyn kann in der Prä- und Perimenopause sowohl in frühem Stadium (Adjuvanz) als auch im metastasierten Stadium verordnet werden. Weitere Informationen bei:

Publicis Vital PR, Anja Dörner

Tel. +49 221 912719-21

E-mail anja.doerner@publicis-vital-pr.de
Sanofi-Synthelabo GmbH. Antworten auf Fragen zum Thema Darmkrebs erhalten Patienten mit der neuen Broschüre «Diagnose Darmkrebs - Ein Ratgeber für Patienten und Angehörige». Vorgestellt werden einzelne Untersuchungsmethoden sowie Therapiemöglichkeiten. Die Broschüre bietet zudem einen Leitfaden für ein Gespräch beim Arzt. Weiterführende Informationen sowie zahlreiche Links und Adressen bietet www.darmkrebstherapie.de.

Weitere Informationen bei:

medical relations $\mathrm{GmbH}$, Eszter Viragh

Tel. +49 21 739769-0, Fax -49

E-mail eszter.viragh@medical-relations.de
Pfrimmer Nutricia GmbH. Mit dem Produkt FortiCare $^{\mathrm{TM}}$ bringt die Erlanger Pfrimmer Nutricia eine Trinknahrung auf den Markt, die speziell für onkologische Patienten, insbesondere mit Tumorkachexie, entwickelt wurde. Durch die hochkonzentrierten Inhaltsstoffe, die patientengerechte Portionsgröße und den in Tests belegten angenehmen Geschmack ist die Nahrung auf die Bedürfnisse von Krebspatienten abgestimmt.

Weitere Informationen bei:

Birke I Partner GmbH, Meike Hallbaue

Tel. +49 9131 8842-16, Fax -44

E-mail meike.hallbauer@birke.de 